\title{
Modification of the Absorption Edge of GaAs Arising from Hot-Electron Effects
}

\author{
McGroddy, J. C.; Christensen, Ove
}

Published in:

Physical Review B

Link to article, DOI:

10.1103/PhysRevB.8.5592

Publication date:

1973

Document Version

Publisher's PDF, also known as Version of record

Link back to DTU Orbit

Citation (APA):

McGroddy, J. C., \& Christensen, O. (1973). Modification of the Absorption Edge of GaAs Arising from HotElectron Effects. Physical Review B, 8(12), 5592-5596. https://doi.org/10.1103/PhysRevB.8.5592

\section{General rights}

Copyright and moral rights for the publications made accessible in the public portal are retained by the authors and/or other copyright owners and it is a condition of accessing publications that users recognise and abide by the legal requirements associated with these rights.

- Users may download and print one copy of any publication from the public portal for the purpose of private study or research.

- You may not further distribute the material or use it for any profit-making activity or commercial gain

- You may freely distribute the URL identifying the publication in the public portal

If you believe that this document breaches copyright please contact us providing details, and we will remove access to the work immediately and investigate your claim. 


\title{
Modification of the Absorption Edge of GaAs Arising from Hot-Electron Effects
}

\author{
J. C. McGroddy and Ove Christensen \\ IBM T. J. Watson Research Center,* Yorktown Heights, New York 10598 \\ Technical University of Denmark, Lyngby, Denmark
}

(Received 25 April 1973)

\begin{abstract}
We have observed a large enhancement of the electric-field-induced optical absorption arising from hot-electron effects in $n$-type GaAs at $77 \mathrm{~K}$. The magnitude and field dependence of the enhancement can be approximately accounted for by a theory attributing the effect to broadening of the final states of the optical transitions by interaction with the nonequilibrium optical phonons produced by the hot electrons.
\end{abstract}

The effect of an electric field on the absorption edge of semiconductors (Franz-Keldysh effect) has been studied rather thoroughly in recent years. ${ }^{1,2}$ Experimentally this phenomenon is normally studied either in insulating crystals or in conducting crystals with blocking contacts so that no current flows, and there are no hot-carrier effects on the absorption edge. In GaAs the influence of hot electrons on the photoluminescence ${ }^{3}$ has been studied, and modulation of the absorption edge by travelling high-field Gunn domains ${ }^{4}$ and by acoustoelectric domains ${ }^{5}$ have also been reported.

In this paper we present some experimental results on hot-electron-enhanced optical absorption in $n-\mathrm{GaAs}$ at $77 \mathrm{~K}$ under conditions where neither the Gunn effect nor acoustoelectric domains are present, so that the results are more amenable to analysis. Experimental results were obtained for photon energies $1.45<\hbar \omega<1.49 \mathrm{eV}$ and electric fields $E \leqslant 2 \mathrm{kV} / \mathrm{cm}$ in samples with electron concentrations $n$ in the range $(1-2) \times 10^{15} \mathrm{~cm}^{-3}$. The field-induced absorption in these samples is found to be much larger than that due to the FranzKeldysh effect.

We discuss the experimental results in terms of two mechanisms by which hot-electron effects can broaden the absorption edge-first directly, by modifying the exciton lifetime via electron-exciton collisions, and second, indirectly, by increasing the collision broadening of both exciton and freeelectron states because of interaction with a perturbed ("hot") optical-phonon distribution.

The experimental results are in reasonable agreement with the calculated results for the hotphonon mechanism with an assumed lifetime of $10^{-11} \mathrm{sec}$ for long-wavelength longitudinal-optical (LO) phonons.

\section{EXPERIMENTAL}

The samples used for the study of the electricfield-induced absorption were cut from single crystals, and had electrically active regions with dimensions $2 \times 1 \times 0.5 \mathrm{~mm}$, the latter being the optical-path length. The contacts were formed by ai- loying with $\mathrm{Au}-\mathrm{Ni}-\mathrm{Ge}$ alloy, and the contact area was always larger than the sample cross-sectional area. The experiments were performed with the samples immersed in boiling liquid $\mathrm{N}_{2}$, and the carrier concentration at $77 \mathrm{~K}$ was in the range $(1-2) \times 10^{15} \mathrm{~cm}^{-3}$. The low-field mobility at $77 \mathrm{~K}$ was typically 20000 . The applied voltage was always kept well below the Gunn threshold, and the $I-V$ characteristics of the samples used showed no anomalies indicative of grossly inhomogeneous field distributions. $40-\mathrm{nsec}$ pulses from a $10-\Omega$ source-impedance pulse generator were applied at the rate of approximately 100 pulses $/ \mathrm{sec}$. The pulses were short enough to avoid any acoustoelectric effects, and the sample temperature rise during an individual pulse was less than $1 \mathrm{~K}$. The light source was a 50-W sungun, and the transmitted light was analyzed with a single-pass-prism monochromator and photomultiplier. The signal was amplified and detected with a boxcar integrator. An integration time of $\sim 100 \mathrm{sec}$ at each discrete wavelength was necessary to achieve adequate signal-to-noise ratio.

\section{RESULTS}

Figure 1 shows the spectral variation of the excess absorption coefficient in the presence of a field of $1.75 \mathrm{kV} / \mathrm{cm}$ for a sample with $n=2 \times 10^{15}$ $\mathrm{cm}^{-3}$ at $77 \mathrm{~K}$, and equivalent data for a sample which is insulating at $77 \mathrm{~K}$. The zero-field absorption for the conducting crystal is also shown. The insulating crystal has a nearly identical zerofield absorption coefficient.

Both the zero-field absorption and the field-induced absorption in the conducting sample in this spectral region fit reasonably well to an experimental dependence of the form $e^{n \omega / \sigma}$, where $\sigma$ has the value $\sim 8 \mathrm{meV}$. The relative change in the absorption coefficient for the conducting sample in Fig. 1 is $\Delta \alpha / \alpha_{0} \simeq 0$.09. Experimentally at $77 \mathrm{~K}$ it is found that as a function of electric field $E$ in conducting samples $\Delta \alpha$ varies as $E^{1.5}$ for fixed photon energy, and is proportional to the electron concentration $n$ over the limited range studied. At 
room temperatures the field-induced absorption in the conducting sample is at least an order of magnitude smaller than at $77 \mathrm{~K}$. By varying the gating of the boxcar integrator relative to the applied pulse it was possible to show that the increased absorption occurs essentially simultaneously (within 7 nsec) with the applied pulse, and does not persist after the pulse is turned off. No dependence of the effect on the direction of polarization of the light relative to the direction of the electric field could be detected.

\section{DISCUSSION}

As can be seen from Fig. 1 the observed fieldinduced absorption in the conducting crystals is much larger than in an insulating sample with essentially the same absorption-edge structure. Comparison of our experimental data with the reductions of the theory of the Franz-Keldysh effect ${ }^{6}$ shows an even bigger discrepancy-i.e., the magnitude of the calculated $\Delta \alpha$ from the theory is smaller than that observed experimentally in the insulating crystal. From the above, and the fact that the effect is proportional to electron concentration in the limited range studied, we conclude that the observed excess $\Delta_{\alpha}$ is due to the presence of free carriers.

\section{THEORY}

\section{General}

In order to make a comparison with real crystals, in which the "ideal" optical absorption, including excitonic effects, is inhomogeneously broadened by microfields due to crystalline imperfections, ${ }^{7}$ we have taken a simplistic view of the effect of additional lifetime broadening due to scattering from the final states of the optical transitions near the absorption edge. Suppose that for a photon energy $\hbar \omega$ the final state involved in the optical transition suffers lifetime broadening with lifetime $\tau(\hbar(\omega)$ because of an additional perturbation-e.g., hot-electron effects. Calling $\Delta \Gamma(\hbar \omega)$ $=\hbar / 2 \tau(\hbar \omega)$, we assume that the absorption in the presence of the perturbation is given by a Lorentzian broadening of the unperturbed absorption, including any inhomogeneous broadening, i.e., from the experimental unperturbed absorption coeff $i$ cient $\alpha_{0}(\hbar \omega)$, as

$$
\alpha(\hbar \omega)=\frac{1}{\pi} \int_{0}^{\infty} \frac{\alpha_{0}\left(\hbar \omega^{\prime}\right) \Delta \Gamma\left(\hbar \omega^{\prime}\right) d\left(\hbar \omega^{\prime}\right)}{\left(\hbar \omega-\hbar \omega^{\prime}\right)^{2}+\left[\Delta \Gamma\left(\hbar \omega^{\prime}\right)\right]^{2}} .
$$

Thus the problem of calculating the absorption induced by an external perturbation, $\Delta \alpha(\hbar \omega)=\alpha(\hbar \omega)$ $-\alpha_{0}(\hbar \omega)$, is reduced to two separate problems.

(i) For all the final states of the optical transitions contributing to $\alpha(\hbar \omega)$, the broadening $\Delta \Gamma(\hbar \omega)$ due to the external perturbation must be calculated.

(ii) $\alpha_{0}(\hbar \omega)$ must be separated into components

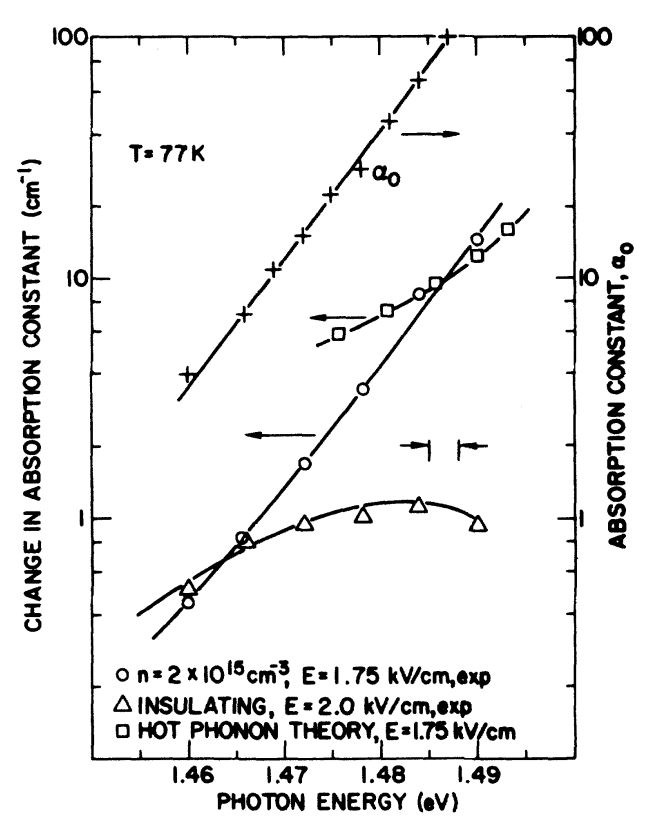

FIG. 1. Field-induced change in absorption constant in conducting (circles) and insulating (triangles) samples and the theoretical result for the perturbed LO-phonon distribution (squares). The upmost curve shows the unmodulated absorption constant, with the background absorption of $14 \mathrm{~cm}^{-1}$ subtracted.

due to different types of final states, and (1) applied to $\alpha_{0}$ with the correct $\Delta \Gamma(\hbar \omega)$ for each type of final state.

In our case (i) essentially means calculating (a) the broadening of the $n=1$ exciton due to elastic and inelastic collisions with electrons and (b) the broadening of both exciton and free-particle states due to inelastic collisions with the LO-phonon distribution function, perturbed by hot-electron-emitted phonons.

\section{Calculation of $\Gamma(\hbar \omega)$}

In our approximate calculations of $\Gamma(\hbar \omega)$ for both types of broadening, we have assumed a drifted Maxwell-Boltzmann distribution function for the hot electrons

$$
\begin{aligned}
& f(k)=\frac{N}{\pi^{3 / 2} k_{0}^{3}} e^{-\left(\overrightarrow{\mathrm{x}}-\overrightarrow{\mathrm{k}}_{D}\right)^{2} / \mathrm{k}_{0}^{2}}, \\
& k_{0}^{2}=2 m^{*} K T^{*} / \hbar^{2},
\end{aligned}
$$

here $h k_{D} / m^{*}$ is the mean electron drift velocity determined from the experimental values of mobility under high-field conditions, $N$ is the electron density. $T^{*}$ is the effective temperature of the hot electrons and was adjusted at each value of the electric field so that the energy-loss rate to emission of LO phonons was equal to the experimental power input.

The two types of broadening considered were: 
(i) elastic collisions and inelastic (both ionizing $1 s-$ continuum and $1 s-2 p$ ) collisions between electrons and excitons, and (ii) inelastic collisions between LO phonons (produced by the hot electrons) and electrons, both free and in exciton states. For each mechanism, the result of the calculation is an electric field dependent $\Delta \Gamma(\hbar \omega)$, which can be used in (1) to determine $\Delta \alpha$.

Electron-Exciton Collisions

Collisions between hot electrons and excitons are of two types-elastic and inelastic. While the detailed variation of the cross section for the various types of collisions is not known, one can scale from the cross section for collisions between electrons and the hydrogen atom. ${ }^{8}$ For both types of collisions, the resulting broadening is

$$
\Gamma=\frac{\hbar}{2 V} \int d^{3} \overrightarrow{\mathrm{k}} f(\overrightarrow{\mathrm{k}})|\overrightarrow{\mathrm{v}}(\overrightarrow{\mathrm{k}})| \sigma(\epsilon(\overrightarrow{\mathrm{k}})),
$$

where $V$ is the crystal volume, $f(\vec{k})$ is the hot-electron distribution function, $\vec{v}(\vec{k})$ is the velocity of an electron with wave number $\vec{k}$, and $\sigma(\epsilon)$ is the energy-dependent cross section for collisions. $\Delta \Gamma$ is then given as the difference between $\Gamma$ calculated with the distribution function $f(\vec{k})$ appropriate to a particular electric field value minus $\Gamma$ calculated for an equilibrium $f(\vec{k})$.

(i) Elastic collisions. Scaling from the hydrogen atom gives the approximate values ${ }^{9}$

$$
\sigma_{e 1}= \begin{cases}20 a_{0} / k & , \quad k a_{0}<0.5 \\ 45 \pi a_{0}^{2} e^{-2.2 k a_{0}}, & k a_{0}>0.5,\end{cases}
$$

where $a_{0}$ is the exciton Bohr radius, taken to be $10^{-8} \mathrm{~m}$, and $k$ is the electron wave number. Since $\sigma_{e 1}$ is a decreasing function of $k$, heating the electron distribution has the effect at $77 \mathrm{~K}$ of reducing $\Gamma_{e 1}$, the broadening due to elastic collisions, below its thermal equilibrium value.

(ii) Inelastic collisions. The important processes for inelastic collisions are transitions from the exciton ground state to the $2 p$ state and to the continuum states. The cross section for both of these processes for energies not too far above threshold can be approximated by ${ }^{8}$

$$
\begin{aligned}
& \sigma_{1 s-\cos t}= \begin{cases}0, & \epsilon<\epsilon_{0} \\
\pi a_{0}^{2}, & \epsilon>\epsilon_{0},\end{cases} \\
& \sigma_{1 s-2 p}= \begin{cases}0, & \epsilon<\frac{3}{4} \epsilon_{0} \\
\pi a_{0}^{2}, & \epsilon>\frac{3}{4} \epsilon_{0},\end{cases}
\end{aligned}
$$

where $\epsilon$ is the electron energy and $\epsilon_{0}$ is the exciton rydberg, taken to be $4 \mathrm{meV}$. At $77 \mathrm{~K}$ these inelastic processes give a contribution $\Gamma_{\text {lnel }}$ which increases when an electric field is applied, which tends to cancel the effects of elastic collisions.

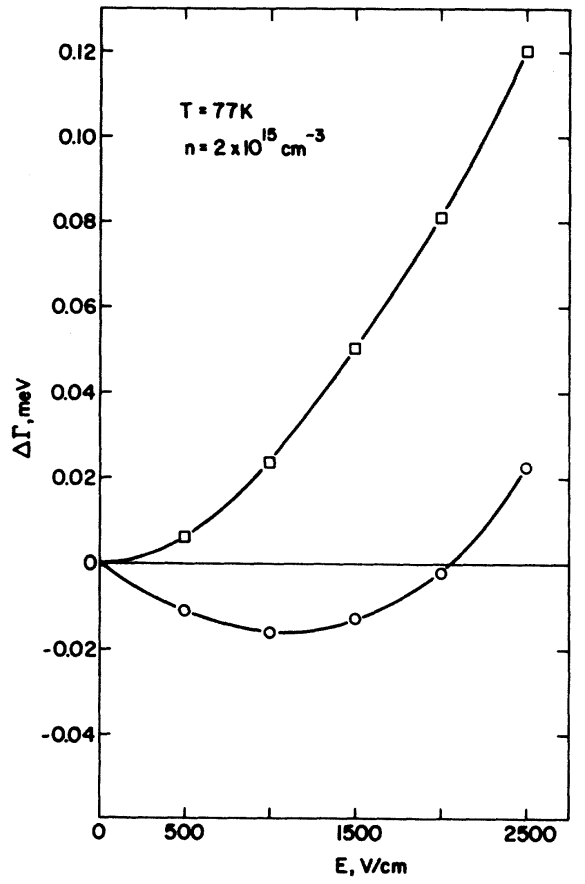

FIG. 2. Field-induced broadening as a function of applied field. The upper curve is the result for the perturbed LO-phonon distribution with $\tau_{\mathrm{LO}}=10^{-11} \mathrm{sec}$, calculated for an electron $50 \mathrm{meV}$ above the band minimum. The lower curve is the sum of elastic and inelastic contributions to the broadening of the exciton ground state by collisions with electrons.

Figure 2 shows the total broadening due to electron-exciton collisions (circles, lower curve) as a function of applied field. To a first approximation there is a cancellation between the elastic and inelastic contributions, although for fields up to about $2 \mathrm{kV} / \mathrm{cm}$ the net result for our model is a reduction in the total broadening. As we see later, the contribution from inelastic collisions of optical phonons with excitions is considerably larger for reasonable long-wavelength-optical-phonon lifetimes.

\section{Optical Phonon Effects}

Under hot-electron conditions the distribution function of the LO phonons is perturbed. That this perturbation can lead to a field enhanced optical absorption below the edge was pointed out by Dumke. ${ }^{10}$ Figure 3 shows some typical spherically averaged LO-phonon distribution functions, calculated using the drifted Maxwellian distribution function for the electrons and the usual matrix element for electron-LO-phonon coupling and a lifetime of $10^{-11}$ sec for the LO phonons. ${ }^{11}$ The perturbation $\delta N(q)$ to the distribution function calculated in this way is 


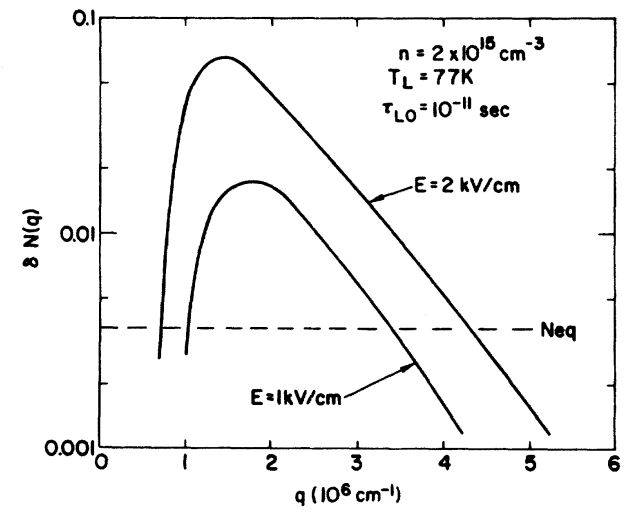

FIG. 3. Spherically averaged perturbation of the LOphonon distribution function due to hot-electron effects, for two field values. The LO-phonon lifetime $\tau_{\text {Lo }}$ was taken to be $10^{-11} \mathrm{sec}$. The dashed curve shows the equilibrium population of these modes which should be added to $\delta N(q)$ to obtain the total $N(q)$ at a given field value.

$$
\begin{aligned}
\delta N(q)= & \frac{(\pi)^{1 / 2} e^{2} \omega_{0} \tau_{L_{0}} m^{*} N}{2 \hbar^{2} q^{3} k_{0} k_{D} \epsilon_{0}}\left(\frac{1}{\kappa_{\infty}}-\frac{1}{\kappa_{0}}\right) \\
& \times \int_{k_{\min }}^{\infty} d k e^{-\left(k^{2}+k_{D}^{2}\right) / k_{0}^{2}}\left(e^{2 k k_{D} / k_{0}^{2}}-e^{-2 k k_{D} / k_{0}^{2}}\right),
\end{aligned}
$$

where $\omega_{0}$ is the LO frequency, $\tau_{\text {LO }}$ is the LO lifetime, $\epsilon_{0}$ is the permittivity of free space, and $\kappa_{\infty}$ and $\kappa_{0}$ are the high- and low-frequency dielectric constants, respectively, and

$$
k_{\min }=m^{*} \omega_{0} / \hbar q+\frac{1}{2} q .
$$

The broadening of an electron state $k$ is then obtained by calculating the additional scattering induced by $\delta N(q)$.

It has the form

$$
\Delta \Gamma_{\mathrm{LO}}(k)=\frac{\text { const }}{k} \int_{a_{\min }}^{a_{\max }} \frac{\delta N(q)}{q} d q .
$$

\section{INDUCED ABSORPTION}

The $\Delta \Gamma$ values for electron-exciton collisions are far smaller than those due to the perturbation of the phonon distribution. In fact, for our particular model, the calculations indicate that for most of the field range studied experimentally the effect of heating the electron distribution in our model is to reduce the broadening parameter $\Gamma$ below its thermal equilibrium value, owing to the inverse dependence of the elastic cross section on electron energy used in Eq. (4).

On the other hand, the $\Delta \Gamma$ values for the hotphonon effect are much larger. Using the values of $\Delta \Gamma$ calculated numerically from Eq. (8) in Eq. (1), we can calculate the change in absorption due to this mechanism. In such a convolution one obtains contributions from energies $\hbar \omega^{\prime}$ reasonably far from $\hbar \omega$ when the absorption coefficient is increasing so rapidly with absorption coefficient as in our case. Since we do not have absorption data on our crystals over a sufficiently wide energy range, we have used the data of Sturge ${ }^{12}$ in performing the convolutions. The two main contributions to $\alpha(\hbar \omega)$ came from the region around $\omega^{\prime}=\omega$ and the region of peak absorption. Above the peak, the integrand in Eq. (1) decreases rapidly due both to the denominator and to the energy dependence of $\Delta \Gamma$.

The result for the calculated $\Delta \alpha(\hbar \omega)$ using Eq. (7) and the calculated $\Delta \Gamma(\hbar \omega)$ over the region studied experimentally is shown as the square points in Fig. 1 for an optical-phonon lifetime $\tau_{\text {LO }}$ of $10^{-11} \mathrm{sec}$. Although the dependence of $\Delta \alpha$ on photon energy is less steep than observed experimentally, the magnitude of $\Delta \alpha$ is in reasonable agreement with experiment at the higher photon energies. In Fig. 4 we show the calculated dependence of $\Delta \alpha$ on electric field for a photon energy of $1.490 \mathrm{eV}$, where theory and experiment agree reasonably well in Fig. 1. The predicted dependence of $\Delta \alpha$ on applied field is rather good.

\section{CONCLUSIONS}

In the temperature region around $77 \mathrm{~K}$, the enhanced electroabsorption under hot-electron conditions is dominated by the selectively increased

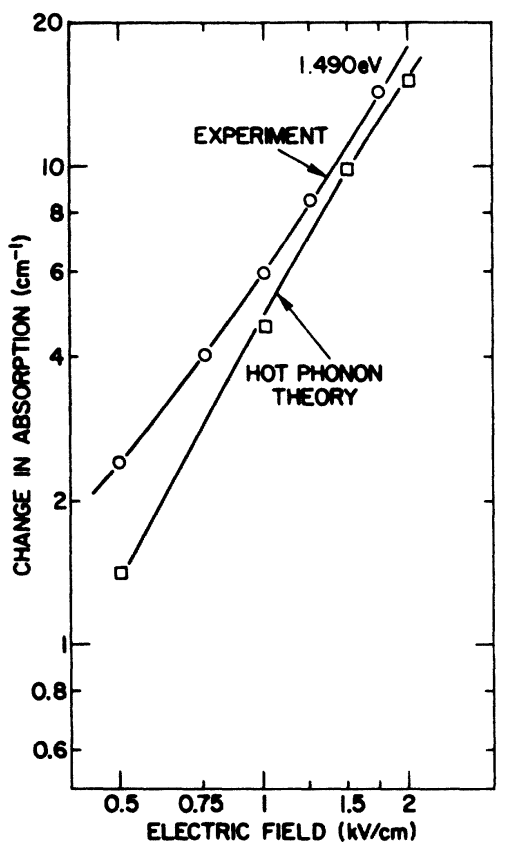

FIG. 4. Field dependence of the induced absorption at a photon energy of $1.490 \mathrm{eV}$. The circles give the experimental result and the squares give the results calculated from the perturbed LO-phonon distribution. Carrier concentration $=2 \times 10^{15} \mathrm{~cm}^{-3}$. 
population of the LO-phonon modes. The effects of elastic and inelastic collisions between electrons and excitons have opposite contributions as a function of electric field, and tend to cancel one another. It would be of considerable interest to study such effects on purer materials at lower temperatures, where exciton peaks are completely re-

*Present address.

${ }^{1}$ T. S. Moss, J. Appl. Phys. 32, 2136 (1961).

${ }^{2}$ E. G. S. Paige and H. D. Rees, Phys. Rev. Lett. 16, 444 (1966).

${ }^{3}$ P. D. Southgate and D. S. Hall, Appl. Phys. Lett. 16, 280 (1970); P. D. Southgate, D. S. Hall, and A. B. Dreeben, J. Appl. Phys. 42, 2868 (1971).

${ }^{4}$ P. Guetin, J. Appl. Phys. 40, 4114 (1969).

'D. L. Spears and R. Bray, Appl. Phys. Lett. 12, 118 (1968).

${ }^{6}$ D. Rees, J. Phys. Chem. Solids 29, 143 (1968). solved, in order to understand the scattering of electrons and phonons with excitons in more detail.

\section{ACKNOWLEDGMENTS}

We acknowledge the assistance of $\mathrm{H}$. Elberg and F. R. Feigel in fabricating samples. The material was kindly supplied by J. Woodall.

7J. D. Dow and D. Redfield, Phys. Rev. B 5, 594 (1972)

${ }^{8}$ H. S. W. Massey and E. H. S. Burhop, Electronic and Ionic Impact Phenomena (Clarendon, Oxford, England, 1969), p. 471.

${ }^{9}$ R. C. C. Leite, J. Shah, and J. P. Gordon, Phys. Rev. Lett. 23, 1332 (1969).

${ }^{10}$ W. P. Dumke, Phys. Rev. 108, 1419 (1957).

${ }^{11}$ A. Mooradian and G. Wright, Solid State Commun. 4, 431 (1966).

${ }^{12}$ M. D. Sturge, Phys. Rev. 127, 768 (1962). 